\title{
Educação permanente em central de regulação: nota prévia
}

\author{
Permanent education in regulation center: prior note \\ Educación permanente en el centro de regulación: nota previa
}

Recebido: 25/09/2021 | Revisado: 01/10/2021 | Aceito: 04/10/2021 | Publicado: 09/10/2021

\author{
Carina Corrêa Bonates Campos \\ ORCID: https://orcid.org/0000-0001-5909-6164 \\ Universidade Federal Fluminense, Brasil \\ E-mail: carina.bonates@gmail.com \\ Elaine Antunes Cortez \\ ORCID: https://orcid.org/0000-0003-3912-9648 \\ Universidade Federal Fluminense, Brasil \\ E-mail: nanicortez@hotmail.com
}

\begin{abstract}
Resumo
Este estudo tem por objetivo informar sobre a execução de um estudo que investiga como a implementação da Educação Permanente na equipe multiprofissional atuante em Central de Regulação pode contribuir para o processo regulatório do SUS, em um município do Rio de Janeiro. Propõe assim, a construção coletiva de estratégias educativas com a finalidade de reorganizar o processo de trabalho deste setor. A pesquisa terá como método de estudo descritivo e exploratório, com abordagem qualitativa, do tipo de pesquisa Convergente Assistencial, buscando articular conhecimento prático e acadêmico. A coleta de dados será dividida em duas etapas: a primeira será desenvolvida através de um questionário semiestruturado com a finalidade de estimular a reflexão e discussão do tema e gerar a problematização necessária para a realização da segunda etapa que consistirá na realização de encontros com a equipe multiprofissional através de rodas de conversa, seguidas de oficinas, utilizando-se de metodologias ativas. A análise dos dados ocorrerá através da análise de conteúdo de Bardin nos questionários e posteriormente terão as rodas de conversa e oficinas analisadas baseando-se no Arco de Maguerez, ambos em consonância com a Política Nacional de Educação Permanente e por inspiração ao teórico Paulo Freire, considerando a problematização advinda do cotidiano e a relação do sujeito com o trabalho educativo. Vislumbra-se que os profissionais atuantes em Central de Regulação consigam apreender o seu protagonismo como autores na busca por processos de mudanças e melhorias da realidade vivenciada, proporcionando a efetividade do acesso dos usuários aos serviços de saúde do SUS.
\end{abstract}

Palavras-chave: Regulação e fiscalização em saúde; Educação Continuada; Profissionais da saúde.

\begin{abstract}
This study aims to inform about the execution of a study that investigates how the implementation of Permanent Education in the multidisciplinary team working in the Regulation Center can contribute to the regulatory process of the SUS in a city in Rio de Janeiro. Thus, it proposes the collective construction of educational strategies in order to reorganize the work process in this sector. The research will be a descriptive and exploratory study method, with a qualitative approach, of the Convergent Assistance type of research, seeking to articulate practical and academic knowledge. Data collection will be divided into two stages: the first will be developed through a semi-structured questionnaire in order to stimulate reflection and discussion of the topic and generate the necessary problematization for the realization of the second stage, which will consist of meetings with the team multiprofessional through conversation circles, followed by workshops, using active methodologies. Data analysis will occur through Bardin's content analysis in the questionnaires and later will have the conversation circles and workshops analyzed based on the Arco de Maguerez, both in line with the National Policy for Continuing Education and inspired by the theorist Paulo Freire, considering the problems arising from everyday life and the subject's relationship with educational work. It is envisioned that professionals working in the Regulation Center are able to grasp their role as authors in the search for processes of change and improvements in the reality experienced, providing effective user access to SUS health services. Keywords: Regulation and inspection in health; Continuing Education; Health professionals.
\end{abstract}

\section{Resumen}

Este estudio tiene como objetivo informar sobre la ejecución de un estudio que investiga cómo la implementación de la Educación Permanente en el equipo multidisciplinario que trabaja en el Centro de Regulación puede contribuir al proceso regulatorio del SUS en una ciudad de Río de Janeiro. Así, propone la construcción colectiva de estrategias educativas con el fin de reorganizar el proceso de trabajo en este sector. La investigación será un método de estudio descriptivo y exploratorio, con enfoque cualitativo, del tipo de investigación Asistencia Convergente, buscando articular conocimientos prácticos y académicos. La recolección de datos se dividirá en dos etapas: la primera se desarrollará a través de un cuestionario semiestructurado con el fin de estimular la reflexión y discusión del tema y generar la problematización necesaria para la realización de la segunda etapa, que consistirá en encuentros con los equipo 
multiprofesional a través de círculos de conversación, seguido de talleres, utilizando metodologías activas. El análisis de datos se realizará a través del análisis de contenido de Bardin en los cuestionarios y posteriormente se analizarán los círculos de conversación y talleres con base en el Arco de Maguerez, ambos en línea con la Política Nacional de Educación Continuada e inspirados en el teórico Paulo Freire, considerando la problematización que surgen de la vida cotidiana y la relación del sujeto con la labor educativa. Se prevé que los profesionales que laboran en el Centro de Regulación sean capaces de captar su rol de autores en la búsqueda de procesos de cambio y mejoras en la realidad vivida, brindando a los usuarios un acceso efectivo a los servicios de salud del SUS.

Palabras clave: Regulación e inspección en salud; Educación Contínua; Profesionales de la salud.

\section{Introdução}

Dentre as utilizações do termo regulação, a concepção de saúde referenda o princípio do direito ao atendimento das necessidades de saúde da população garantindo seu acesso, ações e serviços ofertados em tempo oportuno e de forma equânime pautados nos princípios doutrinários do Sistema Único de Saúde (VILARINS, 2012).

Para garantir o acesso dos usuários do SUS aos recursos assistenciais, diversas políticas de saúde ao longo dos anos foram implementadas, porém cabe enfatizar que as discussões acerca de definição de papéis foram intensificadas a partir do ano de 2001 com as Normas Operacionais Básicas (NOB) que trouxeram os níveis de gestão no processo regulatório, responsabilizando municípios a nível de atenção básica e à União e os Estados a gestão da média e alta complexidade.

Tendo o crescimento populacional aumentado consideravelmente nos últimos anos é perceptível a necessidade de organização e integração das três esferas de governo para garantir a assistência à saúde e ampliação do acesso aos cidadãos aos serviços o que é corroborado pelos esforços adotados pelo Ministério da Saúde que teve como ação estratégica a ampliação da atenção básica e a regionalização e organização da assistência à saúde (BRASIL, 2004).

Em busca da qualidade da ação, da resposta adequada aos problemas clínicos, da satisfação do usuário e para eliminar desconformidades entre a oferta e a demanda, o Ministério da Saúde estabeleceu através da Portaria $\mathrm{n}^{\circ} 1.559$ de $1^{\circ}$ de agosto de 2008 a Política Nacional de Regulação (BRASIL, 2008) que operacionaliza ações sistemáticas através do monitoramento e avaliação da solicitação de consultas e exames, realizadas por um profissional de saúde, sendo observados além das questões clínicas, a determinação de protocolos estabelecidos para a adequação da assistência caso a caso, contemplando princípios da equidade.

Visto o grande desafio que o SUS enfrenta diariamente, no seu planejamento se enquadra em um arsenal de mecanismos normativos e jurídicos que visam garantir e ampliar o acesso aos serviços na criação de protocolos assistenciais e estruturas operacionalizadoras da regulação do acesso denominadas Complexos Reguladores (BRASIL, 2006) que são constituídos por uma ou mais centrais de regulação, o que exige um quantitativo de profissionais capacitados que atuem em funções de coordenação, regulação, atendimento aos usuários, suporte técnico e manutenção de banco de dados, equipamentos e ambiente físico, com infraestrutura adequada e que atenda às especificidades apresentadas pela ação regulatória realizada.

Não obstante, os problemas encontrados no cotidiano do SUS demonstram que deficiências relacionadas à estrutura física, à gestão e aos recursos humanos, somados à baixa resolubilidade de acesso à assistência nos serviços de atenção primária, geram insatisfação e insegurança dos profissionais e usuários do SUS (BARBOSA, 2016).

Assim, repensar a prática e o cotidiano profissional dos agentes do SUS torna-se imprescindível à medida que políticas estratégicas atuem na formação e desenvolvimento de profissionais tais como a Política Nacional de Educação Permanente em Saúde (BRASIL, 2004) permitindo a problematização no cotidiano do trabalho, considerando as necessidades de saúde dos usuários e da transformação profissional e organizacional.

A atuação dos profissionais de regulação assume grande importância no sentido que os mesmos promovem o elo entre o sistema e os usuários e deve garantir que o acesso aos serviços de saúde seja digno e eficiente, pautado em ações de análise e 
verificação de alternativas das solicitações encaminhadas considerando a necessidade de priorização de casos urgentes (BASTOS et al., 2020)

Desta forma, especialmente no que se refere às Centrais de Regulação, evidencia-se a necessidade de intensificação de estudos a respeito da temática relacionando-os à educação permanente, emergindo assim a delimitação do problema desta pesquisa ao questionar: Como a implementação da educação permanente no processo de trabalho em Central de Regulação pode contribuir para o acesso dos usuários a consultas e exames oferecidos pelo SUS?

Assim, traz como objeto do estudo a Educação Permanente em Central de Regulação como estratégia do processo regulatório do SUS e como objetivo geral: Implementar a Educação Permanente no processo de trabalho em Central de Regulação; e objetivos específicos: Identificar as dificuldades da equipe multiprofissional no seu processo de trabalho quanto ao encaminhamento de usuários para consultas e exames na Central de Regulação; descrever como a equipe multiprofissional atuante no setor compreende suas atribuições individuais e coletivas; analisar a efetividade da utilização de estratégias educativas no processo de reorganização do trabalho em Central de Regulação.

Através deste estudo, pretende-se estimular abordagens a respeito do cotidiano do trabalho em regulação, sugerindo possibilidades de reorganização de processos de trabalho em saúde a partir da educação permanente dos profissionais que são os principais atores e articuladores na consolidação das práticas de saúde e garantia de acesso entre sistema e usuário.

\section{Metodologia}

Este estudo está sendo desenvolvido através de uma Dissertação de Mestrado que pertence ao Programa de Pós graduação do Mestrado Profissional Ensino em Saúde (MPES/UFF), possuindo anuência da academia e da unidade pesquisada. Consiste em uma pesquisa com método de estudo descritivo e exploratório, com abordagem qualitativa, do tipo de pesquisa convergente assistencial, a qual busca no cotidiano a problematização necessária para a construção de estratégias e soluções para sua resolução, no contexto da prática vivenciada pelos profissionais, tendo necessariamente o envolvimento ativo destes para proporcionar a transformação da realidade (Trentini, 2014).

Têm por pretensão absorver os diferentes profissionais, em todos os níveis de atuação, pertencentes à equipe multiprofissional e atuantes em Central de Regulação a fim de considerar diferentes concepções sobre a realidade vivenciada no trabalho e no cotidiano ao proporcionar acesso aos serviços de saúde aos usuários do SUS.

Este cenário conta com 15 funcionários compondo uma equipe multiprofissional entre médico, enfermeiros, assistente social, assistente administrativo, técnicos de enfermagem, estagiários e auxiliares de serviços gerais em desvio de função. Desta forma, serão convidados a participar da pesquisa todos os profissionais acima descritos que possuam ao menos 6 meses de experiência com regulação e que aceitem formalmente participar da pesquisa. Excluem-se os profissionais impossibilitados de participar por motivos de férias e licenças em alguma das etapas da coleta de dados.

Para a coleta de dados inicialmente será aplicado um questionário semiestruturado em meio físico para respostas manuais com perguntas abertas e fechadas que estimularão a reflexão e discussão inicial para posteriormente avançarmos para a segunda etapa que consistirá na realização de 3 encontros com a equipe multiprofissional através de rodas de conversa seguidas de oficinas, utilizando-se de metodologia ativa, onde inicialmente será retomada a reflexão inicial obtida pelo questionário e para introduzir nos participantes o conceito proposto pela Educação Permanente relacionando-os às respostas dos questionários e, posteriormente com as oficinas será proposto a construção coletiva de ações educativas que pretendam resolver os problemas / situações encontrados no cenário laboral e discutidas durante as rodas de conversa.

Os participantes da pesquisa assinarão o TCLE concordando em sua participação, voluntária e sem ônus para os mesmos e para a unidade. A previsão de duração de cada encontro será de 1 hora e serão registradas em anotações, fotografias e filmagem. 
Enfatiza-se que as etapas se correspondem onde serão realizadas rodas de conversas seguidas de oficinas e possuirão o objetivo de promover aos participantes uma discussão multidisciplinar, num conjunto de experiências e trocas vivenciadas no dia a dia laboral. Para as diferentes situações de aprendizado, elas se relacionam com experiências anteriores vivenciadas pelos atores sociais com a visualização do contexto prático (FELIPE, 2006).

Os questionários serão analisados sob perspectiva de conteúdo de Bardin (2011), permitindo apontar os problemas existentes no cotidiano de trabalho a serem analisados, discutidos e utilizados como reflexão nas rodas de conversa e oficinas realizadas na etapa dois. Posteriormente, os dados levantados durante as oficinas, serão discutidos a luz da Política Nacional de Educação Permanente em Saúde (PNEPS) e a partir da teoria do dialógica de Paulo Freire.

Conforme a Resolução do Conselho Nacional de Saúde nº 466/2012, esta pesquisa cumprirá todas as especificações legais e éticas provenientes de pesquisa envolvendo seres humanos e foi encaminhada via Plataforma Brasil ao Comitê de Ética em Pesquisa (CEP) do Hospital Universitário Antônio Pedro (HUAP/UFF).

\section{Resultados e Discussão}

Ao propor o uso da EPS como estratégia educativa, espera-se promover o processo reflexivo partindo da identificação dos problemas laborativos cotidianos e, assim, poder melhorar a qualidade da assistência prestada aos usuários do SUS.

Através deste estudo será possível fomentar a participação multiprofissional na construção de estratégias para utilização em seu local de trabalho, propondo artifícios individuais e coletivos de construção e aprendizado relacionados aos serviços oferecidos pelo SUS na Central de Regulação e assim garantir que a população tenha acesso aos mesmos. Tudo isso, corrobora com os objetivos instituídos pela Política Nacional de Educação Permanente, fonte de inspiração para criação desta pesquisa, no qual promove a formação e desenvolvimento dos trabalhadores no SUS, a partir de problemas cotidianos referentes à atenção à saúde e à organização do trabalho.

O estudo é baseado na necessidade de se conhecer com mais especificidade o processo de trabalho em Central de Regulação e, como a educação permanente dos profissionais atuantes pode contribuir para a construção de estratégias e melhorias no processo regulatório do SUS através da educação no trabalho. Portanto, vislumbra-se que as mudanças geradas no processo de trabalho, obtidas por meio de ações educativas, sejam uma das formas mais efetivas para a melhoria do processo regulatório para consultas e exames.

Espera-se que ocorra a incorporação do ensino-aprendizagem à vida cotidiana dos profissionais, assim beneficiando o foco nas reais necessidades da prática de trabalho, favorecendo a população, as políticas públicas de saúde, o ensino e a pesquisa.

\section{Referências}

Brasil. (2002). Ministério da Saúde. Portaria MS/GM n. ${ }^{\circ} 373$, de 27 de fevereiro de 2002 e regulamentação complementar. Brasília, 2002. Dispõe em aprovar, na forma do Anexo desta Portaria, a Norma Operacional da Assistência à Saúde - NOASSUS 01/2002 que amplia as responsabilidades dos municípios na Atenção Básica; estabelece o processo de regionalização como estratégia de hierarquização dos serviços de saúde e de busca de maior equidade; cria mecanismos para o fortalecimento da capacidade de gestão do Sistema Único de Saúde e procede à atualização dos critérios de habilitação de estados e municípios. Brasília, DF, 2002

Brasil. (2004). Ministério da Saúde. MS/SE/DAD/Coordenação-Geral de Apoio à Implementação de Políticas de Saúde/Núcleo de Coordenação da Avaliação de Desempenho do SUS. Oficina sobre Avaliação de Desempenho do SUS. Relatório Final. http://portal.saude.gov.br/portal/arquivos/pdf/documento\%20politica.pdf.

Brasil. (2004). Ministério da Saúde. Portaria nº 198, de 13 fevereiro de 2004. Institui a Política Nacional de Educação Permanente em Saúde como estratégia do Sistema Único de Saúde para a formação e o desenvolvimento de trabalhadores para o setor e de outras providências. Brasília. Ministério da Saúde: https://bvsms.saude.gov.br/bvs/publicacoes/politica_nacional_educacao_permanente_saude_fortalecimento.pdf

Brasil. (2006). Ministério da Saúde. Diretrizes para a implantação de Complexos Reguladores / Ministério da Saúde, Secretaria de Atenção à Saúde, Departamento de Regulação, Avaliação e Controle de Sistemas. Ministério da https://bvsms.saude.gov.br/bvs/publicacoes/DiretrizesImplantComplexosReg2811.pdf 
Brasil. (2012). Ministério da Saúde. Conselho Nacional de Saúde. Resolução No466 de 12 de dezembro de 2012. Dispõe sobre diretrizes e normas regulamentadoras de pesquisas envolvendo seres humanos. Ministério da Saúde: https://bvsms.saude.gov.br/bvs/saudelegis/cns/2013/res0466_12_12_2012.html

Barbosa, D. V. S, Barbosa, N. B. Najberg, E. Regulação em Saúde: desafios à governança do SUS. Cadernos Saúde Coletiva, v. 24, n. 1, p. 49-54, 11 mar. 2016.

Bardin, L. (2011). Análise de conteúdo. Lisboa: Edições 70.

Colombo, A. A., \& Berbel, N. A. N. (2007). A metodologia da problematização com arco de Maguerez e sua relação com os saberes dos professores. Semina: Ciências Sociais e Humanas, 28(2), 121-146. doi: 10.5433/1679-0383.2007v28n2p121

Fernandes, F. C, Cortez, E. A., Laprovita, D, Almeida, L. P., Ferreira, A. F. \& Corvino M. P. F. (2017). Educação permanente em saúde sob a perspectiva de Agostinho de Hipona. Revista Brasileira de Enfermagem, 70(3), 684-689. doi: 10.1590/0034-7167-2016-0484

Feurwerker, L. C. M. (2014). Micropolítica e saúde: produção do cuidado, gestão e formação. Porto Alegre: Rede UNIDA. http://www.saude.sp.gov.br/resources/humanizacao/biblioteca/dissertacoes-e-teses/micropolitica_e_saude_laura_camargo.pdf

González, F. R. Pesquisa qualitativa e subjetividade: os processos de construção da Informação. Tradução de Marcel Aristides Ferrada Silva. São Paulo: Cengage Learning, 2017.

Laprovita, D. (2017). Núcleo de educação em urgência na lógica da educação permanente: serviço de atendimento pré-hospitalar móvel (Dissertação de Mestrado). Escola de Enfermagem Aurora de Afonso Costa, Universidade Federal Fluminense, Niterói. https://app.uff.br/riuff/handle/1/3110

Loro, M. M., \& Zeitoune, R. C. G. (2017). Collective strategy for facing occupational risks of a nursing team. Revista de Escola de Enfermagem da USP, 51, e03205. doi: 10.1590/S1980-220X2015027403205

Merhy, E. E. (2015). Educação permanente em movimento - uma política de reconhecimento e cooperação, ativando os encontros do cotidiano no mundo do trabalho em saúde, questões para os gestores, trabalhadores e quem mais quiser se ver nisso. Saúde em Redes, 1(1), 07-14. doi: 10.18310/24464813.2015v1n1p07-14

Oliveira, F. M. C. S. N., Ferreira, E. C., Rufino, N. A., \& Santos, M. S. S. (2011). Educação permanente e qualidade da assistência à saúde: aprendizagem significativa no trabalho da enfermagem. Aquichan, 11(1), 48-65. from http://www.scielo.org.co/pdf/aqui/v11n1/v11n1a05.pdf

Piazza, M., Ramos, B. J., Oliveira, S. N., Prado, M. L., Massaroli, A, \& Zamprogna, K. M. (2015). Educação permanente em unidades de pronto atendimento 24 horas: necessidade e contribuição à enfermagem. Journal of Nursing and Health, 5(1), 47-54. https://periodicos.ufpel.edu.br/ojs2/index.php/enfermagem/article/view/5408

Prado, M. L., Velho, M. B., Espíndola, D. S., Sobrinho, S. H., \& Backes, V. M. S. (2012). Arco de Charles Maguerez: refletindo estratégias de metodologia ativa na formação de profissionais de saúde. Escola Anna Nery: Revista de Enfermagem, 16(1), 172-177. doi: 10.1590/S1414-81452012000100023

Trentini, M. (2014). Pesquisa Convergente Assistencial-PCA: delineamento provocador de mudanças nas práticas de saúde. Porto Alegre: Moriá.

Vilarins G, Shimizu H, Gutierrez M. A regulação em saúde: aspectos conceituais e operacionais. Saúde em Debate. 2012;36(95):640-47. 